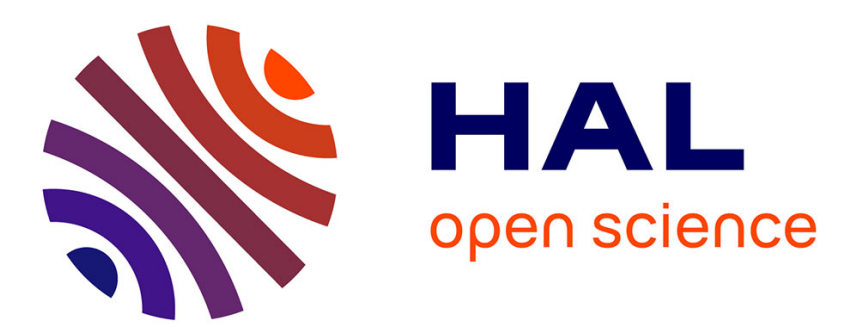

\title{
Adaptive AR and Neurofuzzy Approaches: Access to Cerebral Particle Signatures
}

\author{
Denis Kouamé, Mathieu Biard, Jean-Marc Girault, Aurore Bleuzen
}

\section{To cite this version:}

Denis Kouamé, Mathieu Biard, Jean-Marc Girault, Aurore Bleuzen. Adaptive AR and Neurofuzzy Approaches: Access to Cerebral Particle Signatures. IEEE Transactions on Information Technology in Biomedicine, 2006, 10 (3), pp.559-566. 10.1109/TITB.2005.862463 . hal-01076710

\section{HAL Id: hal-01076710 https://hal.science/hal-01076710}

Submitted on 24 Oct 2014

HAL is a multi-disciplinary open access archive for the deposit and dissemination of scientific research documents, whether they are published or not. The documents may come from teaching and research institutions in France or abroad, or from public or private research centers.
L'archive ouverte pluridisciplinaire HAL, est destinée au dépôt et à la diffusion de documents scientifiques de niveau recherche, publiés ou non, émanant des établissements d'enseignement et de recherche français ou étrangers, des laboratoires publics ou privés. 


\title{
Adaptive AR and Neuro-fuzzy approaches : access to cerebral particle signatures
}

\author{
Denis Kouamé, Member, IEEE, Mathieu Biard, Jean-Marc Girault, Member, IEEE, and Aurore Bleuzen
}

\begin{abstract}
In recent years, a relationship has been suggested between the occurrence of cerebral embolism and stroke. Ultrasound has therefore become essential in the detection of emboli when monitoring cerebral vascular disorders and forms part of ultrasound brain imaging techniques. Such detection is based on investigating the middle cerebral artery using a TransCranial Doppler (TCD) system, and analyzing the Doppler signal of the embolism. Most of the emboli detected in practical experiments are large emboli because their signatures are easy to recognize in the TCD signal. However, detection of small emboli remains a challenge. Various approaches have been proposed to solve the problem, ranging from the exclusive use of expert human knowledge to automated collection of signal parameters. Many studies have recently been performed using time-frequency distributions and classical parameter modeling for automatic detection of emboli. It has been shown that Autoregressive (AR) modeling associated with a abrupt change detection technique is one of the best methods for detection of micro-emboli. One alternative to this technique is a technique based on taking account of expert knowledge. This study aims to unite these two approaches using AR modeling and expert knowledge through a neuro-fuzzy approach. The originality of this approach lies in combining these two techniques and then proposing a parameter referred to as score ranging from 0 to 1 . Unlike classical techniques, this score is not only a measure of confidence of detection but it is also a tool enabling the final detection of the presence or absence of micro-emboli to be performed by the practitioner. Finally, this report provides performance evaluation and comparison with an automated technique,i.e Autoregressive (AR) modeling used in vitro.
\end{abstract}

Index Terms - AR, emboli, Doppler, Detection, False alarm, Model, Neuro-Fuzzy, Non detection, score.

\section{INTRODUCTION}

$\mathbf{S}$ TROKEis the third leading cause of death and morbidity in the western world. In some countries the burden of stroke alone is estimated to be not less than 3 billion dollars per annum. The major factor in the total cost of stroke is the degree of related disability. Thus successful strategies to reduce the impact of stroke through early diagnosis will not only improve the quality of life of patients afflicted with stroke, but will also be very cost effective in many countries.

TransCranial Doppler (TCD) monitoring of signals from microemboli, a sophisticated technique for differentiation of stroke etiology and for early detection of impending stroke in carotid endarterectomy, plays an important role in primary and secondary stroke prevention.

Manuscript received February , 2005; revised October, 2005. This work was supported by European Community (UMEDS Project) and French Government.

The authors are with LUSSI FRE CNRS 2448 University of Tours, 10 boulevard Tonnellé F37032 Tours Cedex France. E-mail: kouame@univtours.fr
An embolus or foreign particle freely moving in the blood stream can be at the origin of sudden obstruction of an artery. This is referred to as an embolism. The consequences of cerebral embolism may be particularly severe, including cerebral infarction. Depending on its origin, an embolus can be a red blood cells aggregate or a piece of fat, a gas bubble, or any other foreign body, carried by the blood stream.

Detection of micro-emboli (small size emboli) and other emboli is important for several reasons such as preventing cerebrovascular accidents, finding the cause of embolism and validating the effectiveness of treatment. The main technique used to detect emboli is the recording of a transcranial ultrasound Doppler signal from the cerebral artery [1]. The embolism signature in the blood stream is then assumed to be a non-predicted high intensity transient signal (HITS) superimposed on the Doppler signal backscattered by the blood. Most existing detection systems use an intensity measurement via the classical Fourier spectrogram, or any other time frequency distribution [2].

Commonly used embolus detection methods consist of comparing the ratio between blood energy distribution and that of the embolus with an empirical threshold [3], [4]. As an embolus crossing the ultrasound beam can be heard, the first emboli detection methods were based on listening to ultrasound Doppler signals. These methods work very well and even outperform the automatic detection technique based on signal power distribution evaluation [5] when dealing with large size (or quite audible) emboli. However, the risk of mistakes due to subjective interpretation in cases of micro-emboli led to investigation of more reliable methods which work when correctly, provided artifacts (which look like an embolus signature) are effectively rejected. Artifact rejection systems consist of either device-related systems (such as multiple gates or a multi-frequency system) or software-related systems, [6], [7].

Standard time-frequency distributions (TFD) were therefore studied for detection; the first being classical short time Fourier analysis which, although simple, is unable to distinguish small emboli. Other TFD techniques such as Wigner Ville distribution and its variants[8], [9], and wavelet transform [10], [11], [12], [13] were also investigated, with limited success due to their intrinsic properties.

However, it has been shown that parametric modeling such as AutoRegressive (AR) modeling, combined with a sudden change detection technique [14], is one of the most reliable approaches to detect micro-emboli automatically. At the same time, it has been shown that integrating human expertise into a detection procedure provides good detection of emboli [15]. 
The common drawbacks of the last two techniques are twofold. First they give rise to a binary decision (existence or absence of embolus in the measurement area), although the embolism phenomenon is still not fully understood by physicians and no other means (e.g ultrasound brain scanner) is simultaneously available to check the reality of what is detected. Secondly they are mutually exclusive, whereas their advantages could be combined in a single approach. Human expertise has been taken into account, for this purpose, by using fuzzy logic. Thus we consider an adaptive fuzzy approach in order to fit the variability of embolus signatures. Some attempts at embolus detection have been made using neural networks [16]. This study only used a classical neural network system such as "black-box", with its classical drawbacks (namely high number of iterations). Moreover, one important question, which was neither specific to the study reported by Kemeny et al [16] nor solved in any automatic embolus detection, is the intrinsic evaluation of the reliability of the detection method. Here, we propose instead the use of both fuzzy logic and the neural network for optimization of membership function via the so-called neuro-fuzzy approach. A particular feature of our investigations is that, in order to take into account the possible uncertainty regarding the event detected and to provide an evaluation of the reliability of the event detected, we introduced a parameter score ranging from 0 to 1 as output of our system. After briefly revisiting the AR technique in the context of micro-embolus detection and introducing adaptive fuzzy modeling, the experimental results are used for comparison between parametric modeling and the fuzzy approach.

\section{BACKGROUND}

\section{A. Doppler signal and parametric autoregressive modeling}

Ultrasound (US) Doppler is the main method used for embolus detection. This technique consists in transmitting by means of a transducer, an ultrasound wave with frequency $f_{0}$, to a selected area of a cerebral artery. After reception and demodulation, the resulting (analytic) complex number Doppler signal, see e.g. [17], denoted here $x(t)$ may be defined by :

$$
x(t)=I(t)+j Q(t)=K(t) \exp \left[j\left(2 \pi f_{d}(t)+\phi(t)\right)\right]
$$

where $j=\sqrt{-1}, K(t)$ is the random magnitude of the Doppler signal depending on the characteristics of the transducers and the measurement area, and $\varphi(t)$ is the random phase depending on the positions of the scatterers of the flowing fluid. The frequency shift $f_{d}$, called Doppler frequency, is proportional to $f_{0}$.

This Doppler signal contains a large amount of information concerning the moving target.

Unlike commonly used methods for embolus detection, parametric autoregressive (AR) modeling consists of working on a model of the signal and not directly on the signal. We briefly recall the classical results below. Considering a discrete time complex Doppler signal $x(n)=x\left(t=n T_{e}\right)$ sampled at frequency $1 / T_{e}$ and assuming that it is the output of a p-order Autoregressive (AR) model, it can be expressed by :

$x(n)=-a_{1}(n) x(n-1)-a_{2}(n) x(n-2)-\ldots-a_{p}(n) x(n-p)+\eta(n)$

where $a_{i}(n)$ are complex coefficients defining the AR model, $p$ is order of the model ( number of coefficients) and $\eta(n)$ is a complex white noise. For convenience, the previous equation is commonly expressed in matrix form as :

$$
x(n)=\varphi^{T}(n) \theta(n)+\eta(n)
$$

where

$$
\begin{gathered}
\varphi^{T}(n)=[-x(n-1), \ldots,-x(n-p)], \text { and } \\
\theta^{T}(n)=\left[a_{1}(n), \ldots, a_{p}(n)\right] .
\end{gathered}
$$

It is well known that modeling the signal $x$ as an AR process thus corresponds to obtaining the vector $\hat{\theta}(n)$ from $x$ which is an estimate of the vector $\theta(n)$. This estimation can be performed using, for example, the Recursive Least Squares (RLS) algorithm. Let

$$
e(n)=x(n)-\hat{x}(n)
$$

be the prediction error. When the model (3) holds, the prediction error tends in probability, to white noise, when the number of observation data extends to infinity. Since the autocorrelation function (AF) of a white noise equals zero at any lag, except for the initial lag $(\mathrm{n}=0)$, the $\mathrm{AF}$ of prediction error therefore provides interesting Decision Information (DI), i.e information containing the embolus signature for this parametric method. Indeed, when an embolus crosses the sample volume, the prediction error will no longer be a white noise, and its AF at lag 1 (for example) will no longer be zero. The $\mathrm{AF}$ at lag 1 can be expressed by:

$$
C_{n} \stackrel{\text { def }}{=} C(1)=\frac{1}{n} \sum_{k=1}^{n} e(k) e(k-1)
$$

This can be estimated recursively each time $n$ using a forgetting factor $\alpha(0<\alpha \leq 1)$ by :

$$
C_{n}=\alpha C_{n-1}+(1-\alpha) e(n) e(n-1)
$$

Here $\alpha=0.9$. Due to the above, $C_{n}$ will be almost zero for a normal Doppler signal, and the presence of an embolus will be characterized by a abrupt change. Therefore, to detect an embolus, we have to construct DI. Thus here :

$$
D I=\left|C_{n}\right|
$$

The probability density function $p(y)$ of this DI is [18] :

$$
p(y)=\frac{1}{\pi} \int_{0}^{\infty} \exp [-|y| \cosh (u)] d u, y \neq 0
$$

An example of an embolus recorded on a patient is shown in fig. 1 to illustrate this.

In order to evaluate the reliability of embolus detection, such detection is performed in the framework of hypothesis testing. 
EMBOLI
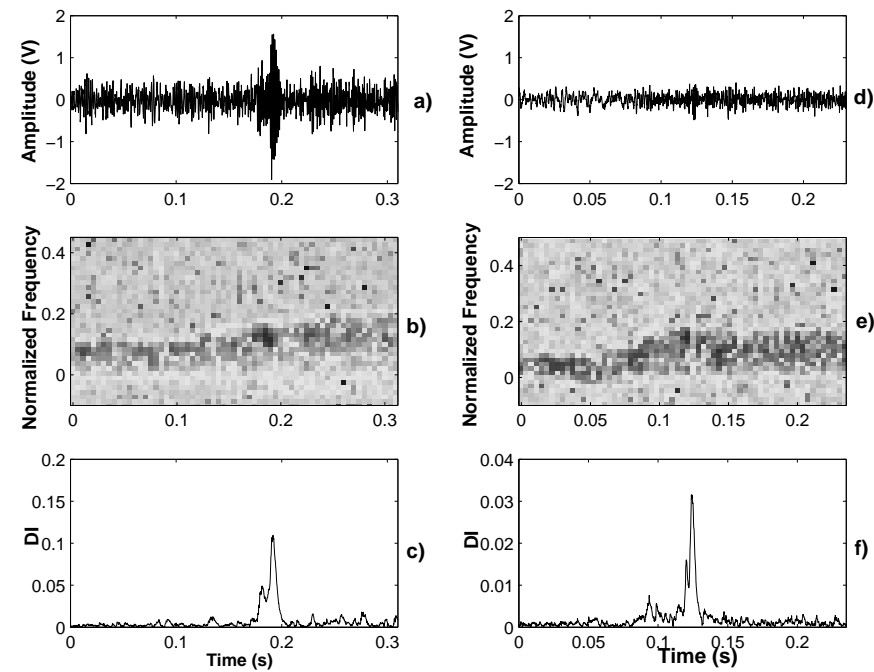

Fig. 1 .

Typical in vivo circulating embolus signatures in symptomatic patients. (a) real part Doppler signal of a large size embolus, (b) and (c) are spectrogram and DI, respectively, (d) real part Doppler signal of a smaller size embolus, $(e)$ and $(f)$ are spectrogram and DI, respectively.

Two hypotheses, i.e. $H_{0}$ and $H_{1}$, representing the absence and the presence of an embolus, respectively, have to be tested. Assuming that the decision made is based on single observations of the process or the received signal represented by random variable $\mathrm{Y}$, and that the possible values of $Y$, constitute the observation set denoted $O$, the set $O$ is then divided into two subsets $\left(O_{0}\right.$ and $\left.O_{1}\right)$ such that if values of $\mathrm{Y}$ belong to $O_{i}$ the decision is $D_{i}$, with $i=0,1$. The probability density functions of $\mathrm{Y}$ corresponding to each hypothesis are denoted $f_{Y \mid H_{0}}\left(y \mid H_{0}\right)$ and $f_{Y \mid H_{1}}\left(y \mid H_{1}\right)$, where $y$ is a particular value of the random variable $Y$. Denoting $P\left(D_{i} \mid H_{j}\right)$, the probability of deciding $D_{i}$ when $H_{j}$ is true, it follows that,

$$
P_{i j} \stackrel{\text { def }}{=} P\left(D_{i} \mid H_{j}\right)=\int_{O_{i}} f_{Y \mid H_{j}}\left(y \mid H_{j}\right) d y .
$$

With these definitions we have

$$
\begin{gathered}
P F A=P_{10}=1-P_{00} \\
P N D=P_{01}=1-P_{11}
\end{gathered}
$$

where PFA is the probability of false alarm and PND is the probability of non-detection evaluated using eq.(8) and (9). In practice each hypothesis is characterized by decision information (DI), and belonging to $O_{0}$ or $O_{1}$ is represented by a threshold $(\lambda)$. PND and PFA can thus be obtained by inverting eqs.(8) and (9). PFA and PND are measures of reliability.

Finally the well known power spectrum density expression, from eq.(3), denoted $P_{x}(n, f)$ to account for its timedependent behavior, can be obtained for each time $n$ as :

$$
P_{x}(n, f)=\frac{\sigma^{2}}{\left|1+\sum_{k=1}^{p} a_{k}(n) \exp (-2 \pi j f k)\right|^{2}}
$$

where $\sigma^{2}$ is the power of the noise $\eta$ and $f$ is normalized frequency $-0.5 \leq f \leq 0.5$ and $j=\sqrt{-1}$.

\section{B. Neuro-Fuzzy approach}

In this section, we also provide a brief introduction to the concepts used to study a problem with the neuro-fuzzy approach. Introduced in 1965 by Zadeh [19], the fuzzy approach is based on fuzzy reasoning or approximate reasoning, which is an inference procedure used to derive conclusions from a set of fuzzy if-then rules, as follows : "IF conditions THEN conclusion". Conditions and conclusion are of the type :" $\mathrm{x}$ is A", "y is B", respectively and $\mathrm{x}$ and $\mathrm{y}$ are variables representing for example input and output of the system under consideration. A and B are referred to as linguistic terms such as for instance "LARGE", "LOW",... and are characterized by membership functions $\mu_{A}$ and $\mu_{B}$. For a particular value $x_{0}$ of $\mathrm{x}$, it can be said that " $\mathrm{x}$ is $\mathrm{A}$ " with a degree of truth $\mu_{A}\left(x_{0}\right)$. An important characteristic of such a system is that, due to its structure, it is immediately possible to insert human expertise through the rules. Direct use of this fuzzy reasoning may need a high number of cumbersome manual settings in complex problems. Neuro-fuzzy modeling was introduced [20],[21] to account for this and fit the possible change in the system. Using our application, we consider here the case of the Sugeno model. That means that conclusions of rules are crisp linear combinations of variables. Many choices are possible [21]. Our choice is explained below.

For the purpose of illustration, consider the example of two rules (R1 and R2), two inputs $\left(x_{1}\right.$ and $\left.x_{2}\right)$ and one output $(z)$ below.

R1 : IF $x_{1}$ is $A_{11}$ and $x_{2}$ is $A_{12}$ THEN $f_{1}=p_{1} x_{1}+q_{1} x_{2}+r_{1}$ R2 : IF $x_{1}$ is $A_{21}$ and $x_{2}$ is $A_{22}$ THEN $f_{2}=p_{2} x_{1}+q_{2} x_{2}+r_{2}$ The resulting output is $z=\frac{\mu_{1} f_{1}+\mu_{2} f_{2}}{\mu_{1}+\mu_{2}}$; where $\mu_{i}=\mu_{A_{i 1}}\left(x_{1}\right) \times$ $\mu_{A_{i 2}}\left(x_{2}\right)$ with $\mathrm{i}=1,2$, as shown in (fig.2-a).

The symbol $\times$ may be either a product symbol or any other T-norm symbol[19],[20],[21]. T-norm is the operation, performed in order to provide the value of the output membership function value, when different fuzzy values, say $\mu_{i}$, $i=1,2,$. result from different input membership functions (see example below). The T-norm used here is a Min-Max operator. This system can be modeled as a four layer neural network (fig.2-b).

This can be generalized for a system with $\mathrm{N}$ rules as in (fig.3). Thus a general neuro-fuzzy system is an equivalent four layer network (fig.3) for a fuzzy system of which the ith rule is :

$\mathrm{Ri}: \quad$ IF $x_{1}$ is $A_{1 i}$ and $\ldots x_{n}$ is $A_{n i}$ THEN $f_{i}=p_{n i} x_{n}+\ldots+$ $p_{1 i} x_{1}+p_{0 i}$ 

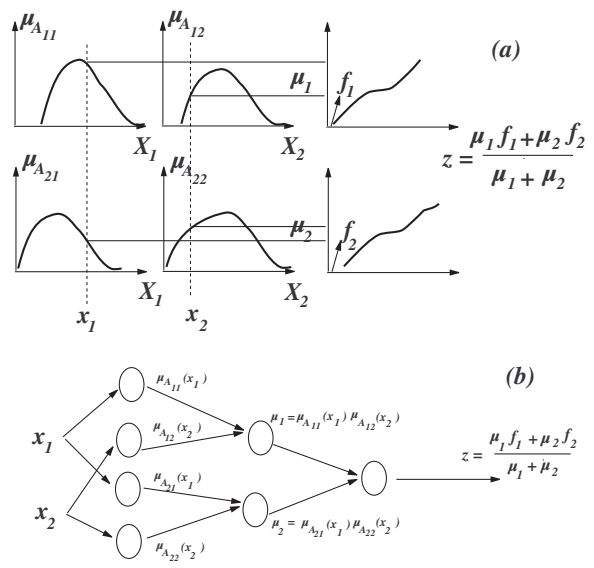

Fig. 2.

Sugeno Fuzzy inference system with two rules (a) and its equivalent neural net model (b).

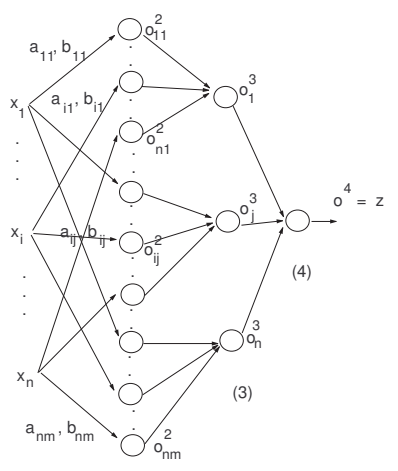

(2)

Fig. 3.

$N$ inputs four layers neural network system.

where " $x_{i}$ is $A_{i j}$ " is evaluated by $\mu_{i j}\left(x_{i}\right) . \mu_{i j}$ is a the membership function which is typically gaussian (of course other types can be used) with mean $a_{i j}$ and variance $b_{i j}$.

Given a set of rules, the neuro-fuzzy technique adjusts the parameters of the system under consideration through the four layers defined as :

Layer 1. This is the input layer. Inputs are $x_{i}, i=1 \ldots n$

Layer 2. Each node corresponds to evaluation of degrees of truth. $o_{i j}^{(2)}=\mu_{i j}\left(x_{i}\right)=\exp \left(-\left(\frac{x_{i}-a_{i} j}{b_{i} j}\right)^{2}\right) ; i=1, \ldots n, j=$ $1, \ldots, m$

Layer 3. Each node performs involvements through the T-norm operation : $o_{j}^{(3)}=\prod_{n=1}^{n} o_{i j}^{(2)} ; i=1, \ldots n, j=1, \ldots, m$

Layer 4. This output node performs defuzzification : $z=$ $\frac{\sum_{j=1}^{m} o_{j}^{(3)} f_{j}}{\sum_{j=1}^{m} o_{j}^{(3)}}$, where $f_{j}$ is the consequent part of the $j^{t h}$ rule $: f_{j}=p_{n j} x_{n}+\ldots+p_{1 j} x_{1}+p_{0 j}$

$a_{i j}$ and $b_{i j}$ are referred to as premise parameters and $p_{i j}$ as consequent parameters. Basically, estimating and adjusting parameters is based on a hybrid algorithm in which the consequent parameters are identified by the least squares method. Many alternative algorithms have been developed to speed up the convergence, [22], [23], [24], [25]. In order to speed up the convergence rate and mostly to overcome the problem of selection of number of rules not solved by these techniques, we used the $\delta$-operator Recursive Least Squares method [26],[27]. The problem of selection of number of rules, which is opened even in the fast algorithm, is solved here by using the matrix decomposition $U D V^{H}$ technique [28].

Due to the complexity of our application, and even if it is certain that the high intensity transient signal (HITS) detected is an embolus signature, in practice, there is no absolute guarantee that it is in fact an embolus signature. This is true both for expert physician and for all automatic detection systems.

To take this into account, we decided to provide a PFA for the parametric method for each HITS detected. For the neuro-fuzzy method, instead of giving a binary decision (absence/presence of embolus) as output of our system we gave a measure of detection, i.e. a score between 0 and 1 .

This simple idea is, to our knowledge, new. With conventional approaches used for embolus detection, it is not possible to provide such an output (which is binary in these conventional approaches). The score output we propose here - a kind of "re-fuzzification" of the output - is both a decision and self evaluation of the decision.

This thus means that a score above 0.7 reveals the presence of an embolus, with a high level of reliability (70\%), and a score below 0.3 reveals absence of embolus. We thus have an uncertainty range between 0.3 and 0.7 which has to be accounted for the physician.

\section{APPLICATION}

In order to validate the above techniques, we used experimental in vitro data obtained using "blood mimicking fluid" (BMF) embedded in a "Tygon" tube and circulated by a pump. Emboli were simulated by acrylic particles of different sizes (diameters from $200 \mu m$ to $400 \mu m$ ). The BMF had acoustic properties similar to those of blood. Doppler signals were recorded using a Transcranial Doppler system, (WAKI 2 from ATYS MEDICAl).

Signals were recorded with the following experimental protocol:

* probe frequency: $2 \mathrm{MHz}$

* Pulse Repetition Frequency $(\mathrm{PRF})=6 \mathrm{KHz}$

* The Tygon tube was located so that explored depth was $54 \mathrm{~mm}$.

* burst length: $8.3 \mathrm{~mm}$

* sample length: $4.59 \mathrm{~mm}$

The inputs of the neuro-fuzzy approach were the estimated neuro-fuzzy parameters and the characteristics of the signal. For example, for a single gate system these characteristics were:

- DI (Decision information, see section II-A), with a range from 0 to $50 \mathrm{~dB}$ above the detection threshold.

- (HITS) duration, (range 0 to $300 \mathrm{~ms}$ ).

- Amaxn/Amaxp, i.e. the ratio between maximum power spectrum density in the domain of negative and positive frequencies, respectively, (range 0 to $50 \mathrm{~dB}$ ). 
- fmaxn, i.e. the normalized frequency of the maximum power spectrum density in the domain of negative frequencies, (range -0.5 to 0 ).

- fmaxp, i.e. the normalized frequency of the maximum power spectrum density in the domain of positive frequencies, (range 0 to 0.5 ).

It is important to note here that all these parameters were computed from the parametric model, section II-A. A gaussian membership function was used for each of these parameters .

The output of the system, in this approach was the score.

The inputs of the parametric method were only the above characteristics of the Doppler signal and the output was the decision.

Here are some additional practical details about our neuro-fuzzy system.

The "expert knowledge" is available knowledge on emboli. This knowledge helps to choose the input parameters and relevant rules. For example most of the artifacts are bi-directional that is, they have frequency components in both positive and negative frequency domains. So inputs "fmaxn" and "fmaxp" were useful for dealing with direction of detected events. Here are the details of our system.

1) We recorded a set of artifact signals (29 in the example shown in fig.4) and another set of acrylic signals (12 in example shown in fig.4).
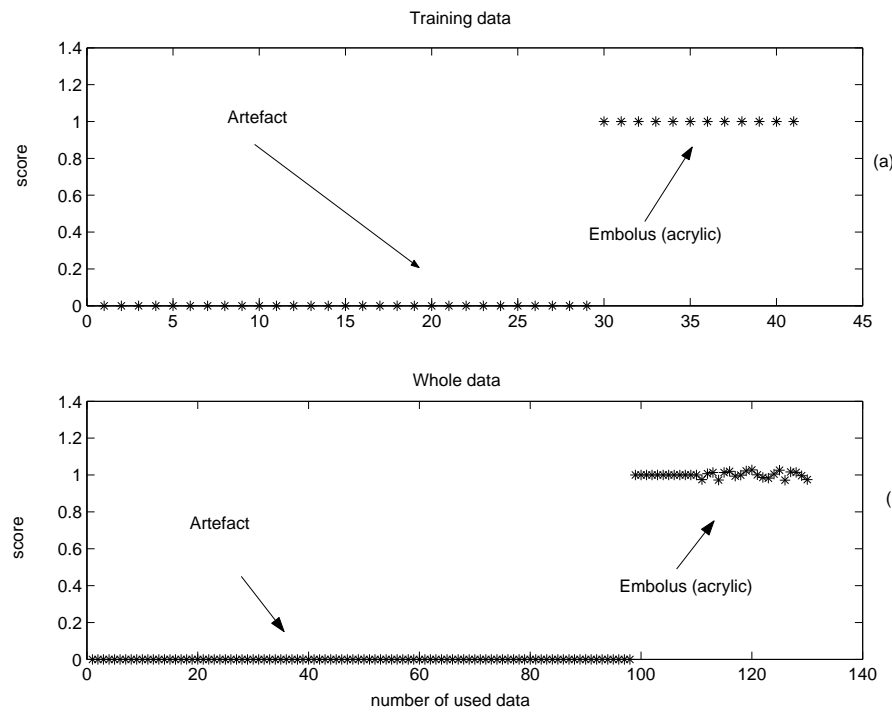

Fig. 4.

Score evaluated using neuro-fuzzy methods. (a) training data (40 signals). (b) Whole data (130 signals).

For each of these 41 signals we had five input parameters and one linear output (score). The artifact signals output scores were set to 0 and those of acrylic were set to 1 . So we had an input matrix $\mathrm{X}$ of $41 \times 5$ values and an output vector $\mathrm{Y}$ of $41 \times 1$ values consisting of relevant zeros and ones. The number of rules per input was estimated. Here we had for DI, HITS duration, Amaxn/Amaxp, fmaxn, fmaxn respectively 3,3,3,2,2.
Then we chose gaussian membership functions. And from the above inputs and outputs, the membership parameters and output parameter were estimated using the hybrid algorithm. For illustration, the membership functions of Input "Amxn/Amaxp" are shown in fig.5.
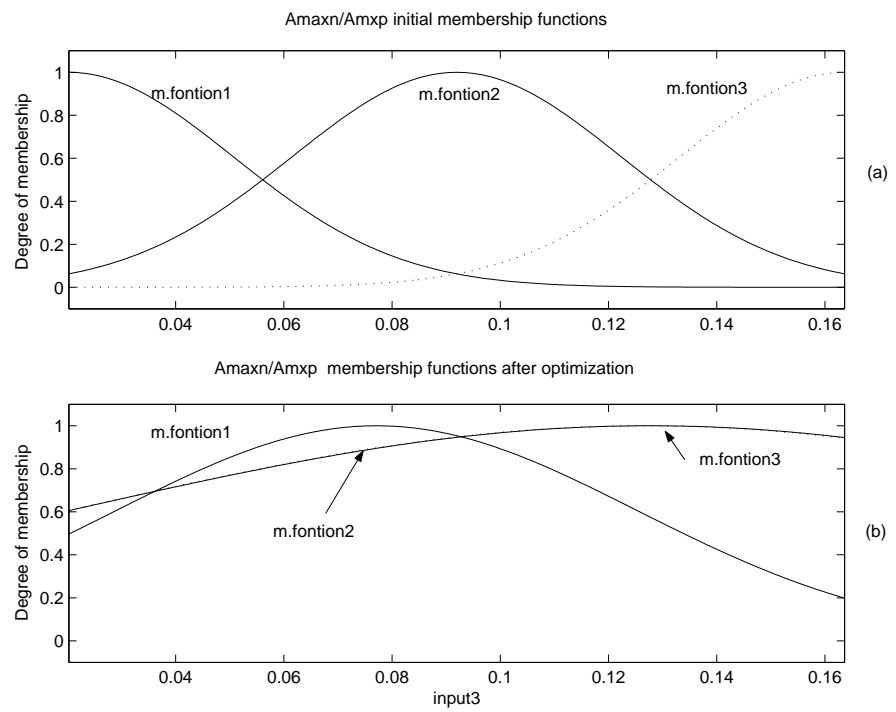

Fig. 5.

Illustration of membership function behavior using Input "Amxn/Amaxp": (a) and (b) are respectively initial membership functions and membership function after optimization. m.function1, m.function2 and m.function 3 are membership function \# 1, 2 and 3

In fig.5 (a) and (b) are shown respectively initial membership functions and membership functions after optimization. When these parameters were estimated and the system was asked to find the scores for the above signals, the answer was of course correct, that is, 0 for artifact and 1 for acrylic signals.

2) Then from the whole data (including the training data), five input parameters per signal are computed, and the relevant scores were estimated.

\section{RESUlTS}

Several signals were recorded consisting of different types of artifact, together with acrylic particles. A typical in vitro circulating acrylic Doppler signal is shown in fig.6, with its relevant DI.

A typical in vivo artifact signal is shown in fig.7.

Two sizes (diameters) of acrylic particle (240 $\mu m$ and $300 \mu \mathrm{m}$ ) were used first.

A set of 130 signals consisting of a mixture of artifacts (slight taps on the transducers) and acrylic particles were 

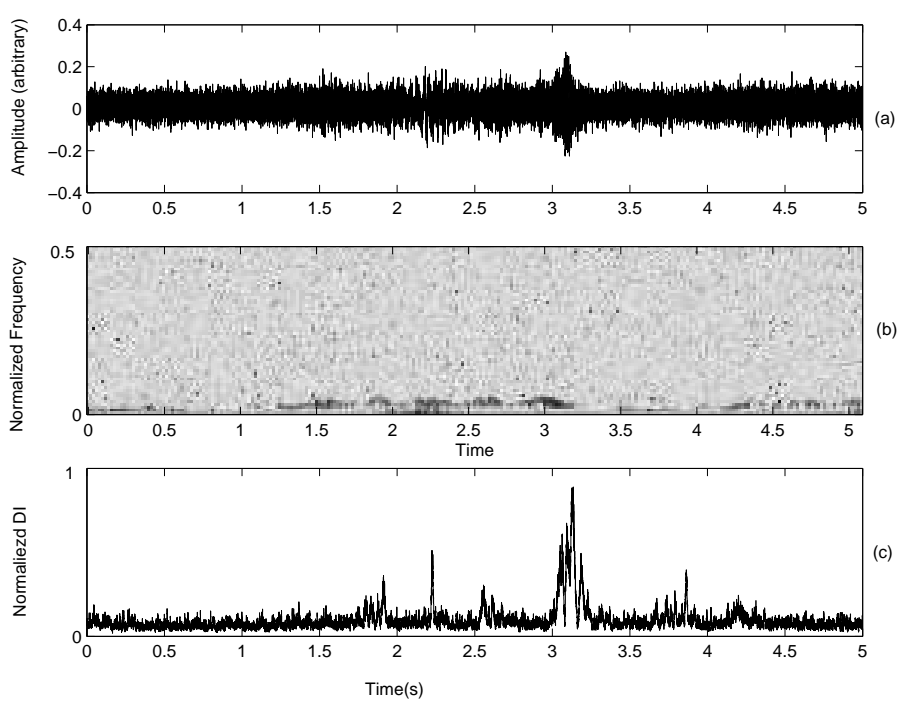

Fig. 6.

Typical in vitro circulating acrylic (of $240 \mu m$ ) signature. (a) real part Doppler signal, (b) spectrogram, (c) DI.
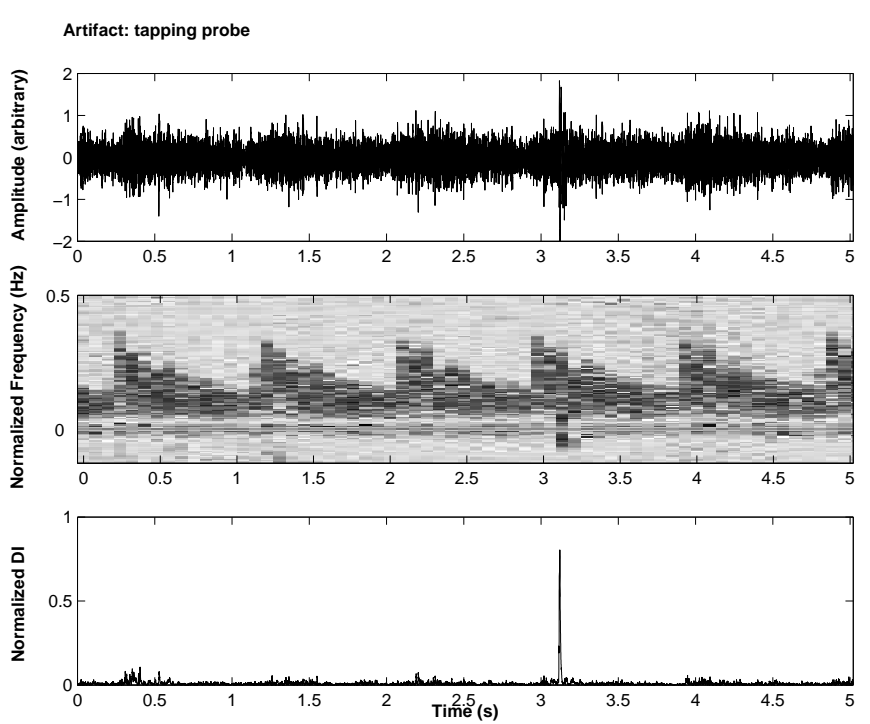

Fig. 7.

Typical in vivo artifact signature. (a) real part Doppler signal, (b) spectrogram, (c) DI.

recorded independently. Forty one signals of this set were used to train the neuro-fuzzy system. Once trained all the 130 signals were used for test. The results are shown in fig.4.

Our system was trained only once throughout. Then to test the generalization of the model learnt, 1,000 independent signals consisting of acrylic particles of different sizes (diameters $200 \mu m, 230 \mu m, 230 \mu m, 260 \mu m, 280 \mu m, 300 \mu m$ ) and artifacts of different types were recorded and presented to the system. The results shown in fig.4 are representative of the different tests made with acrylic particles of different sizes. Artifacts were detected at scores close to zero. The scores for acrylic particles were always higher than 0.7 , except for fewer than $5 \%$ of cases.
We cannot in the strict sense talk about PFA and PND, however for purpose of comparison we defined for neuro-fuzzy approach: $P F A=\frac{N F A}{N R}$ where $N F A$ was number of artifacts detected with score $>0.2$ and $N R$ was the number of tests. $P N D=\frac{N N D}{N R}$ where $N N D$ was number of emboli (acrylic particles) detected with score $<0.7$ and NR was the number of tests.

(b) These led to $P F A \simeq 0$ and $P N D \leq 5 \%$. This is summarized in Table.I.

TABLE I

\begin{tabular}{||c||c|c||}
\hline & PFA & PND \\
\hline \hline $300 \mu \mathrm{m}$ & $0 \%$ & $3 \%$ \\
\hline $280 \mu \mathrm{m}$ & $0 \%$ & $3 \%$ \\
\hline $260 \mu \mathrm{m}$ & $0 \%$ & $3.5 \%$ \\
\hline $240 \mu \mathrm{m}$ & $0 \%$ & $4 \%$ \\
\hline $230 \mu \mathrm{m}$ & $0 \%$ & $4 \%$ \\
\hline $200 \mu \mathrm{m}$ & $0 \%$ & $4.1 \%$ \\
\hline
\end{tabular}

Equivalent Probability of False Alarm(PFA) and Probability of Non-Detection (PND) for neurofuzzy method.

This means that this algorithm missed less than $5 \%$ of the acrylic signal data. In all the cases, when no acrylic was present, the decision provided by the algorithm was correct.

The results for the parametric method are summarized in Table.II.

TABLE II

\begin{tabular}{||c||c|c||}
\hline & PFA & PND \\
\hline \hline $300 \mu \mathrm{m}$ & $4 \%$ & $0 \%$ \\
\hline $280 \mu \mathrm{m}$ & $3.8 \%$ & $0 \%$ \\
\hline $260 \mu \mathrm{m}$ & $4 \%$ & $1 \%$ \\
\hline $240 \mu \mathrm{m}$ & $3.5 \%$ & $6.25 \%$ \\
\hline $230 \mu \mathrm{m}$ & $4 \%$ & $6.5 \%$ \\
\hline $200 \mu \mathrm{m}$ & $4.1 \%$ & $6.7 \%$ \\
\hline
\end{tabular}

Probability of False Alarm(PFA) and Probability of Non-Detection (PND) for parametric method.

PFA was less than $5 \%$ and PND was less than $7 \%$ for a given detection threshold. PFA and PND were defined as follows : $P F A=\frac{N F A}{N R}$ where $N F A$ was number of false alarm obtained for $N R$ tests. $P N D=\frac{N N D}{N R}$ where $N N D$ was number of non detections obtained for $N R$ tests. These estimations of PFA and PND provide results similar to direct computations [18].

This means that the algorithm detected something (acrylic particles) whereas nothing was present in the signal in less than $5 \%$ of the $(1,000)$ data used. This algorithm missed less than $7 \%$ of the acrylic signal data.

It is important to note that, as for most conventional systems, artifact rejection was performed by another procedure. Two main procedures are usually used. The first uses two or more gates (measurement areas), one of which can be located at an area where it is unlikely to detect an embolus. If the event detected is present simultaneously at all the gates, it is an artifact. The second is based on estimating the direction of the detected event. If the detected event is bi-directional then it is an artifact. 
The performance of the two embolus detection techniques presented was very similar. The advantage of our neuro-fuzzy technique was that processing of artifacts was immediate, and only one gate was needed to perform embolus detection and artifact rejection. Thus this detection system does not require additional gates as in conventional detection systems. In vivo validation of this system is now being performed.

\section{CONCLUSION}

A specific neuro-fuzzy approach is proposed here in the framework of embolus detection. This technique was compared to automated embolus detection based on a parametric AR method using in vitro data. Although the performance of the two techniques was similar, the neuro-fuzzy technique presents the advantage of being able to perform detection using only one gate. This technique is thus a promising method to detect emboli effectively with a low cost system. In vivo validation of this system is currently being performed.

\section{ACKNOWLEDGEMENT}

The authors wish to thank anonymous reviewers for their careful readings.

\section{REFERENCES}

[1] M.P. Spencer, G.I. Thomas, S.C. Nicholls, and L.R. Sauvage, "Detection of middle cerebral artery emboli during carotid endarterectomy using transcranial doppler ultrasonography," Stroke, vol. 21, pp. 415-423, 1990.

[2] L. Cohen, "Time-frequency distributions, a review," Proceedings of The IEEE, vol. 77 , no. $7,1989$.

[3] M.A. Moehring and J.R. Keppler, "Pulse doppler ultrasound detection characterization and size estimation of emboli in flowing blood," IEEE Trans. Biomed. Eng., vol. 41, pp. 35-44, 1994.

[4] M.A. Moehring and J.A. Ritcey, "Sizing emboli using pulse doppler ultrasound, part i: Verification of ebr model," IEEE Trans. Biomed. Eng., vol. 43, pp. 572-580, 1996.

[5] Er. V. Van Zuilen, W. H. Mess, C. Jansen, I. Van Der Tweel, Jan Van Gijn, and R. G.A. Ackerstaff, "Automatic embolus detection compared with human experts : A doppler ultrasound study," Stroke, vol. 27, pp. 1840-1843, 1996.

[6] R. Brucher and D. Russell, "Automatic embolus detection and artifact rejection with the first multifrequnecy transcranial doppler," Stroke, vol. 33, pp. 1969-1974, 2002.

[7] G. Devuyst, P.A. Despland, and J. Bougousslavsky, "The matching pursuit : A new method of characterizing microembolic signal," Ultrasound Med. Biol., vol. 26, pp. 1051-1056, 2002.

[8] J.L. Smith, D.H. Evans, L. Fan, A.J. Thrush, and A.R. Naylor, "Processing doppler ultrasound signals from blood-borne emboli," Ultrasound Med. Biol., vol. 20, pp. 455-462, 1994.

[9] E. Roy, P. Abraham, S. Montresor, and J.L. Saumet, "Comparaison of time-frequency estimators for peripheral embolus detection," Ultrasound Med. Biol., vol. 26, pp. 419-423, 2000.

[10] C. Guetbi, D. Kouame, A. Ouahabi, and J.P. Remenieras, "New emboli detection methods," in Proc. IEEE International Ultrasonics Symposium, 1997.

[11] P.W. Lui, B.C. Chan, F.H. Chan, P.W. Poon, H. Wang, and F.K. Lam, "Wavelet analysis of embolic heart sound detected by precordial doppler ultrasound during continuous venous air embolism in dogs," Anesth. Analg., vol. 2, pp. 325-331, 1998.

[12] N. Aydin, S. Padayachee, and H. Markus, "The use of the wavelet transform to describe embolic signals," Ultrasound Med. Biol., vol. 25, pp. 953-958, 1999.

[13] B.S. Krongold, A.M. Sayeed, M.A. Moehring, J.A. Ritcey, M.P. Spencer, and D. L. Jones, "Time-scale detection of microemboli in flowing blood with doppler ultrasound," IEEE Trans Biomed Eng, vol. 46, pp. 10811089, 1999.
[14] J.M. Girault, D. Kouamé, A. Ouahabi, and F. Patat, "Micro-emboli detection:an ultrasound doppler signal processing view point," IEEE Trans. on Biomed. Eng., vol. 47, no. 11, pp. 1431-1438, 2000.

[15] L. Fan, D.H. Evans, and A.R. Naylor, "Automated embolus identification using a rule-based expert system," Ultrasound Med. Biol, vol. 27, no. 8, pp. 1065-1077, 2001.

[16] V.Kemeny, D. W. Droste, S. Hermes, D. G. Nabavi, G. S.-Altedorneburg, M. Siebler, and E. B. Ringelstein, "Automatic embolus detection compared with human experts : A doppler ultrasound study," Stroke, vol. 27, pp. 1840-1843, 1996.

[17] Jensen J.A., Estimation of blood velocities using ultrasound. A signal processing approach, Cambridge : University Press,, 1996.

[18] D. Kouamé, J-M. Girault, A. Ouahabi, and F. Patat, "Reliability evaluation of emboli detection using a statistical approach," in Proc. IEEE Utrason. Sympos., 1999.

[19] L.A. Zadeh, "Fuzzy set," Inform. and Contr., vol. 8, pp. 338-353, 1965.

[20] C.T. Lin and C.S.G. Lee, "Neural-network-based fuzzy logic control and decision system," IEEE Trans. Comput., vol. 40, pp. 1320-1336, 1991.

[21] J.S.R. Jang, "Anfis : Adaptive-network-based fuzzy inference system," IEEE Trans. Syst. Man and Cybern, vol. 23, pp. 665-683, 1993.

[22] M. Onder Efe and Okyay Kaynak, "A novel optimization procedure for training of fuzzy inference systems by combining variable structures systems technique and levenberg marquardt algorithm," Fuzzy Sets and Systems, vol. 122, pp. 153-165, 2001.

[23] M.T. Hagan and M.B. Menhaj, "Training feedforward networks with markardt algorithm," IEEE Trans. Neural Net., vol. 5, pp. 989-993, 1994.

[24] G. Lera and M. Pinzolas, "Neighborhood based levenberg marquardt algorithm for neural network training," IEEE Trans. Neural Net., vol. 13, pp. 1200-1203, 2002.

[25] A.K. Palit and R. Babuska, "Efficient training algorithm for takagisugeno type neuro-fuzzy network," in Proc. The 10th IEEE International Conference on Fuzzy Systems, vol 3, 2001.

[26] D. Kouamé, J.F. Roux, and A. Ouahabi, "Parametric estimation: Improvement of the rls approach using a differential approach," Int. J. of Modelling and Simulation, vol. 19, pp. 18-26, 1999.

[27] D. Kouamé, J.M Girault, V. Labat, and A. Ouahabi, "Delta high order cumulant-based recursive instrumental variable algorithm," IEEE Signal Processing Letters, vol. 7, pp. 262-265, 2000.

[28] S. Niu, D.G. Fisher, and D. Xiao, "A factored form of instrumental variable algorithm," Int. Journ. of . Adapt. Cont. and Sig. Proc, vol. 7, pp. 261-273.

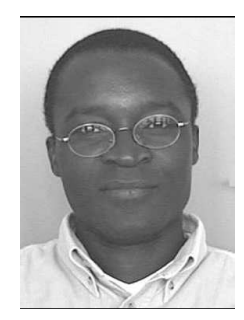

Denis Kouamé Denis Kouamé (M'97) was graduated in computer integrated in manufacturing engineering from the Ecole d'Ingt'enieurs de Tours, Tours, France, in 1992. He received the Ms C in automatic control and computer engineering from the University of Tours, France, followed by the Ph.D. degree in computer engineering and automaticcontrol and signal processing from the University of Tours in 1996. Since 1994 he has been with the G.I.P. Ultrasons-L.U.S.S.I. Currently he is lecturer at the University of Tours. His research interests includes ultrasound signal processing and relevant applications, namely, industrial and biomedical velocimetry and emboli detection.

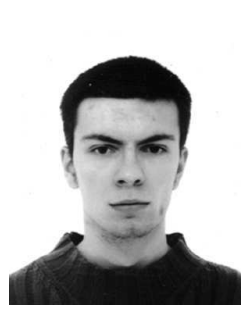

Mathieu Biard Mathieu BIARD was obtained the master's degree in Image Instrumentation, Computing from the University of Dijon, France, in 2001 He received the Ph.D. degree in computer engineering and signal processing (engineering science) from the University of Tours in 2005. He was with the G.I.P. Ultrasons-L.U.S.S.I. Currently, he is employed at the CHU Bretonneau of Tours, (CIT Ultrasons). His research interests includes ultrasound signal processing and relevant applications, like, emboli detection and characterization and, actually breast ultrasound elastography. 


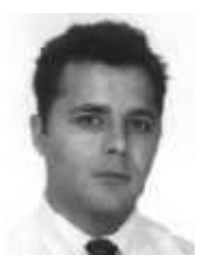

Jean-Marc Girault Jean-Marc Girault (S'98 M'00) received the Master degree of signal processing, biological and medical imaging from the University of Angers, France, in 1996 and the Ph.D. degree in "sciences de l'ingénieur" from the University of Tours. He is lecturer at the University of Tours in signal processing in the I.U.T. of Blois. His research area concerns ultrasound signal processing in biomedical applications and particularly in emboli detection.

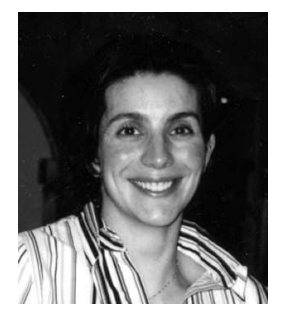

Aurore Bleuzen Aurore Bleuzen received the Ph. $D$. degree in biological and medicine images and signals in 2003 from the University of Tours, France. She works as radiological practitioner in the Technological Innovation Center (CIT) of ultrasound of the hospital center of Tours, France. Her research interest includes the applications of contrast agents in ultrasound, the emboli detection and the development of high resolution ultrasound system. 